\title{
Exchange broadening of EPR line in $\mathrm{ZnO}: \mathrm{Co}$
}

\author{
P. Sati ${ }^{1}$, V. Pashchenko ${ }^{2}$, and A. Stepanov ${ }^{1}$ \\ ${ }^{1}$ Laboratoire Matériaux et Microélectronique de Provence, Case-142 \\ Université Paul Cézanne and CNRS, 13397, Marseille Cedex 20, France \\ E-mail: anatoli.stepanov@L2MP.fr \\ ${ }^{2}$ Physikalisches Institut, Johann Wolfgang Goethe Universität, 1 Max-von-Laue-Strasse \\ Frankfurt am Main 60438, Germany
}

Received March 3, 2007

\begin{abstract}
We study the $X$-band EPR spectra of $\mathrm{Co}^{2+}$ in single crystalline $\mathrm{Zn}_{1-x} \mathrm{Co}_{x} \mathrm{O}(x=0.001-0.075)$ thin films grown by plasma-assisted molecular beam epitaxy. By analyzing the EPR linewidth behavior we argue that the exchange-narrowing model, usually applied to Mn-based II-VI DMS, fails here and that a combined effect of exchange and dipolar broadening can explain the linewidth variation with Co content and temperature.
\end{abstract}

PACS: $76.30 . \mathrm{Fc}$ Iron group $(3 d)$ ions and impurities (Ti-Cu).

Keywords: electron paramagnetic resonance, diluted magnetic semiconductors.

Diluted magnetic semiconductors (DMS), the magnetic properties of which are due to the substitution of cations by transition-metal (TM) ions, have become a focus of considerable interest in recent years as essential materials for practical semiconductor spintronic devices such as spin filters [1] or spin polarizers [2]. The theoretical predictions based on the local spin density approximation (LSDA), triggered extensive studies of $\mathrm{ZnO}$ :TM alloys with a special focus on $\mathrm{ZnO}: \mathrm{Co}$ as the most promising candidate for a room-temperature ferromagnetic (FM) semiconductor [3]. Many experiments have been reported on this material fabricated by a variety of methods [4-6]; however, the magnetic properties of $\mathrm{ZnO}$ :Co still remain a controversial issue since the observed magnetic behavior appears to be strongly dependent on the preparation methods and is poorly reproducible. Ferromagnetism was reported for thin films and bulk samples of $\mathrm{ZnO}$ :Co with a very large spread of spontaneous magnetic moment from $6.1 \mu_{B} /$ Co to $0.01 \mu_{B} /$ Co accompanied by a Curie temperature well above room temperature [4-9]. At the same time the absence of ferromagnetism and paramagnetic behavior down to helium temperatures in $\mathrm{ZnO}$ :Co were claimed by many authors [10-16].

On the theoretical side, LSDA has difficulties when applied to the magnetic state of TM-doped $\mathrm{ZnO}$, since it does not account for correlations between $d$-electrons and leads to a semimetallic FM ground state. Quite surpris- ingly, an improved version of LSDA, LSDA $+U$ also leads to controversial results as regards the exchange constant sign between $\mathrm{Co}^{2+}$ ions in $\mathrm{ZnO}$. Indeed, in their recent paper, Chanier et al. [17] show that the exchange constants, $J^{\text {out }}$ and $J^{\text {in }}$, between nearest-neighbor (NN) $\mathrm{Co}$ ions in the $\mathrm{ZnO}$ wurtzite structure are both negative (AFM) and have the values $-9 \mathrm{~K}$ and $-21 \mathrm{~K}$, respectively. In contrast, Lee and Chang [18] and Sluiter et al. [19] have detected a competition between FM $J^{\text {out }}$ and AFM $J^{\text {in }}$ in Co-doped $\mathrm{ZnO}$.

The EPR offers an interesting alternative to traditional magnetic and optical methods providing complementary information on the electronic properties of transition metals ions. For example, EPR is able to probe the exchange interactions between TM ions by studying the well known phenomenon of the exchange narrowing of an EPR line. In this case a broadened line, say, by the magnetic dipole interaction, $\mathcal{H}_{d}$, is narrowed by a fast dynamics resulting from the exchange coupling, $J$. This gives finally a linewidth $\Delta H \sim \mathcal{H}_{d}^{2} / J$ [20]. Interestingly, the exchange narrowing picture explains the linewidth of various Mn-based II-VI DMS at high temperatures [21]. It was also established, that at low temperatures these DMS exhibit, quite universally, a critical line broadening - a phenomenon which is also driven by the exchange coupling [22-24]. More recently, the exchange-narrowing model was confirmed for $\mathrm{Zn}_{1-x} \mathrm{Mn}_{x} \mathrm{O}$ thin films having various $\mathrm{Mn}$ contents $[25,26]$. To our knowledge, no 
detailed EPR investigations have been yet performed on $\mathrm{Zn}_{1-x} \mathrm{Co}_{x} \mathrm{O}$.

In this paper we report the $X$-band EPR experiments on $\mathrm{Zn}_{1-x} \mathrm{Co}_{x} \mathrm{O}$ single crystalline thin films, performed in a wide range of temperature and Co content, which were undertaken in order to gain more insight into the role played by the magnetic $\mathrm{Co}-\mathrm{Co}$ interaction in $\mathrm{ZnO}$ : Co.

$\mathrm{Zn}_{1-x} \mathrm{Co}_{x} \mathrm{O}$ thin films with $x=0.001-0.075$ were grown on sapphire substrates by plasma-assisted MBE and had thicknesses of about $1 \mu \mathrm{m}$, the $c$-axis of the wurtzite structure being perpendicular to the film plane. The conductivity of the films was $n$-type, with residual carrier concentrations $n_{e}<10^{18} \mathrm{~cm}^{-3}$.

We first discuss the magnetic properties of the studied $\mathrm{ZnO}$ :Co films. The data were obtained using a commercial SQUID magnetometer (Quantum Design MPMS) in magnetic fields up to $50 \mathrm{kOe}$ and in the temperature range 2-300 K. A pure $\mathrm{ZnO}$ film on a sapphire substrate and a sample holder were examined separately and their signals were subsequently subtracted from the total magnetic moment. The Co content $x$ of the studied samples was determined by energy dispersive $\mathrm{x}$-ray (EDX) microanalysis. For the lowest concentration $(x<0.005)$, the $x$ value was determined by magnetic measurements.

In Fig. 1 we show the temperature dependence of the inverse static magnetic susceptibility, $\chi^{-1}(T)$, measured at $H=10 \mathrm{kOe}$ and $H \perp c$ for $x=0.052$.

A linear increase of $\chi^{-1}(T)$ at higher temperatures can be fitted to the Curie-Weiss law $\chi^{-1}(T)=(T-\theta(x)) / C(x)$, with the usual notation for $\theta(x)$ and $C(x)$. Note that the curve in Fig. 1 is representative of many experiments in two respects, namely, i) for all measured samples the $\operatorname{sign}$ of $\theta$ is found to be negative, ii) the continuous increase of $\theta$ with $x$ is also observed.

The inset of Fig. 1 shows the magnetization of the $\mathrm{Zn}_{1-x} \mathrm{Co}_{x} \mathrm{O}$ film with $x=0.052$ as a function of magnetic

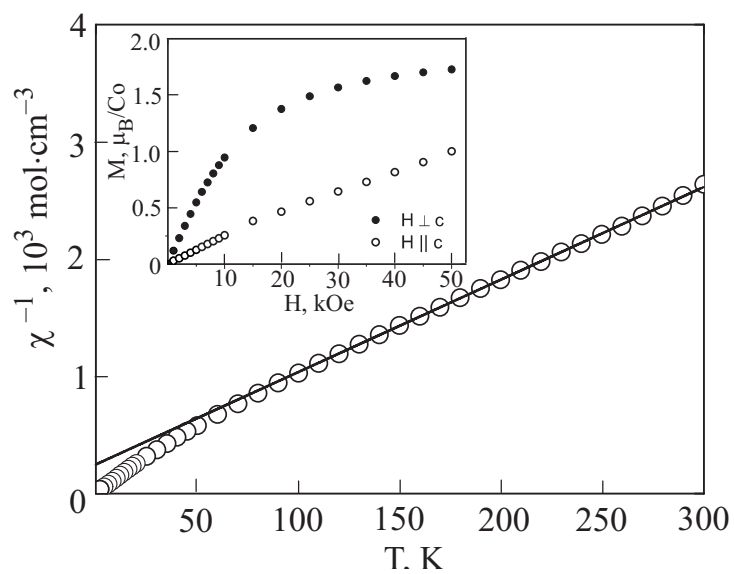

Fig. 1. Temperature dependence of the inverse magnetic susceptibility for a $\mathrm{Zn}_{1-x} \mathrm{Co}_{x} \mathrm{O}$ sample with $x=0.052$ taken at $H=10 \mathrm{kOe}$ and $H \perp c$. The solid line is the Curie-Weiss law. The inset shows $M$ vs $H$ plot for the same sample at $T=2 \mathrm{~K}$ and for $H \| c$ and $H \perp c$. field at $T=2 \mathrm{~K}$. As expected from the results on weakly Co-doped $\mathrm{ZnO}$ films [27], $M(H)$ curves reveal a considerable magnetic anisotropy of $\mathrm{Co}^{2+}$ in the wurtzite lattice. In order to probe the exchange interactions in $\mathrm{ZnO}: \mathrm{Co}$, we have compared the experimental data with the results of simulations in the framework of a cluster model. Our conclusion is that both $\left|J^{\text {in }}\right|$ and $\left|J^{\text {out }}\right|$ exceed $10 \mathrm{~K}$ and are negative in $\mathrm{ZnO}$ : $\mathrm{Co}$ [28].

We turn now to our EPR results. The $X$-band EPR spectra were recorded using a Bruker EMX spectrometer equipped with a standard $\mathrm{TE}_{102}$ cavity and a continuous helium flow cryostat that allows temperature scans between 4 and $300 \mathrm{~K}$. The films with an area of $3 \times 3 \mathrm{~mm}$ were mounted on a quartz rod sample holder. The angle between the $c$-axis of the films and the direction of the static magnetic field $H$ was controlled by a goniometer with a precision better than $\pm 0.25^{\circ}$.

In Fig. 2 we present the low-temperature EPR spectra of $\mathrm{ZnO}: \mathrm{Co}$ samples with different $\mathrm{Co}^{2+}$ concentration measured at $9.4 \mathrm{GHz}$ for $H \| c$. For the lowest $x$ the reso-

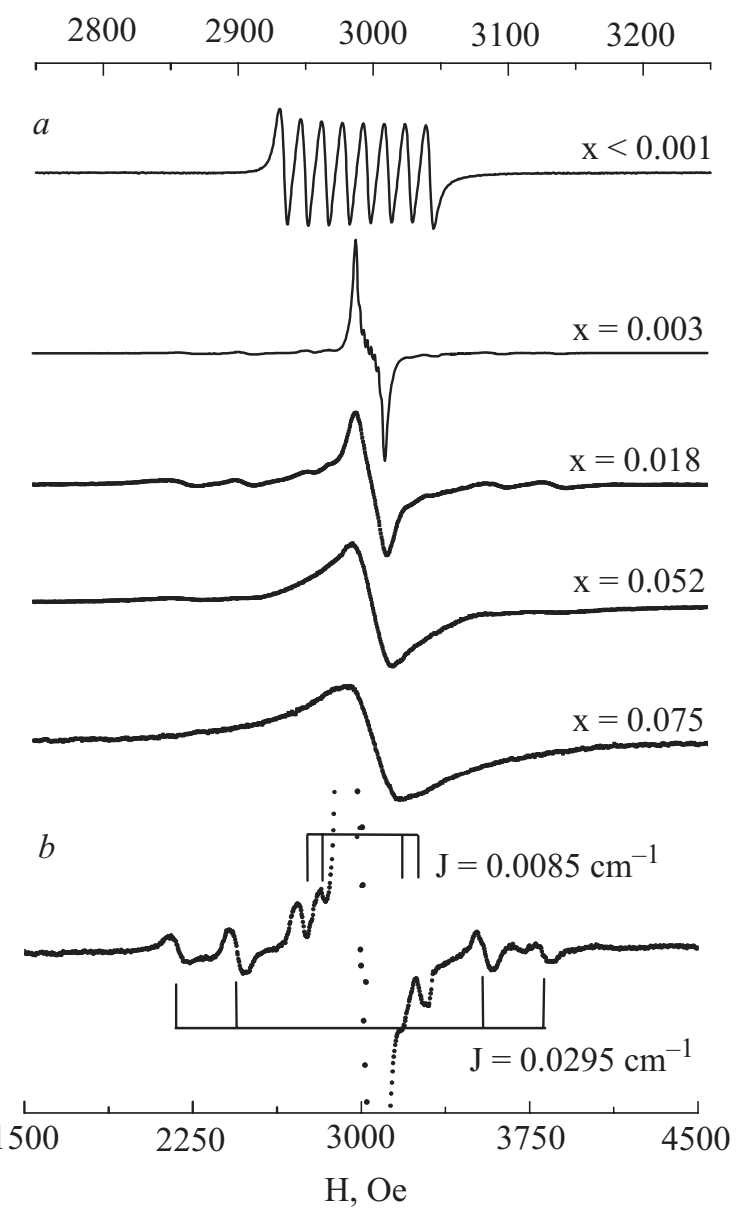

Fig. 2. EPR spectra taken at $7 \mathrm{~K}$ and for $H \| c$ on $\mathrm{Zn}_{1-x} \mathrm{Co}_{x} \mathrm{O}$ films with different Co content. Note that the upper scale corresponds only to the sample with $x<0.001(a)$. EPR spectrum of the sample with $x=0.003$ shown on an enlarged scale. The vertical bars indicate the positions of two exchange pair spectra characterized by two different exchange constants $(b)$. 
nance spectrum consists of eight equally spaced components resulting from the hyperfine interaction of the electron spin of $\mathrm{Co}^{2+}$ and its nuclear spin, $I=7 / 2$. The main EPR spectrum originates from the low-lying doublet $S_{z}= \pm 1 / 2$ of a $S=3 / 2$ ground-state manifold and its position is determined by the following spin Hamiltonian

$$
\mathcal{H}_{\text {spin }}=\mu_{B} g_{\|} H_{z} S_{z}+D S_{z}^{2}+A_{\|} S_{z} I_{z},
$$

where $g_{\|}=2.236, D=2.76 \mathrm{~cm}^{-1}$ and $A_{\|}=16.1 \cdot 10^{-4} \mathrm{~cm}^{-1}$ were inferred from experiments for the lowest $x$ $[27,29,30]$. Additionally EPR spectra of $\mathrm{Co}^{2+}$ exchange pairs are observed and shown in detail in Fig. 2, b. (Details of the pairs spectra interpretation will be published in our forthcoming paper.) With increasing of the Co concentration the observed hyperfine structure (HFS) first becomes poorly resolved, for $x=0.003$, and then completely disappears for $x>0.02$ due to a line broadening. This line broadening is our main concern here.

In order to separate the linewidth caused by the HFS from the total one we used the following procedure. The observed EPR spectra were modeled by eight $(2 I+1)$ Lorentzian components corresponding to the HFS transitions. The resonance fields of the eight Lorentzian were fixed by the position of the center of gravity of the hyperfine octet in accordance with the observed $g$-factor. Then, we calculate the individual position of each component using $A_{\|}$. The full width at half maximum, $\Delta H$, of individual HFS components were adjusted to get the best agreement with the recorded signal.

Fig. 3 shows the linewidth obtained in this way as a function of both Co content and temperature. As it may be seen from the inset of Fig. 3, $\Delta H$ grows almost linearly with $x$. This is in significant disagreement with previously reported data on Mn-doped DMS. In this latter case and at low $x<0.1$ a rapid decrease of $\Delta H$ is observed suggesting the exchange narrowing of an initially broadened line. Another important feature of the observed linewidth behavior is its temperature dependence shown in Fig. 3.

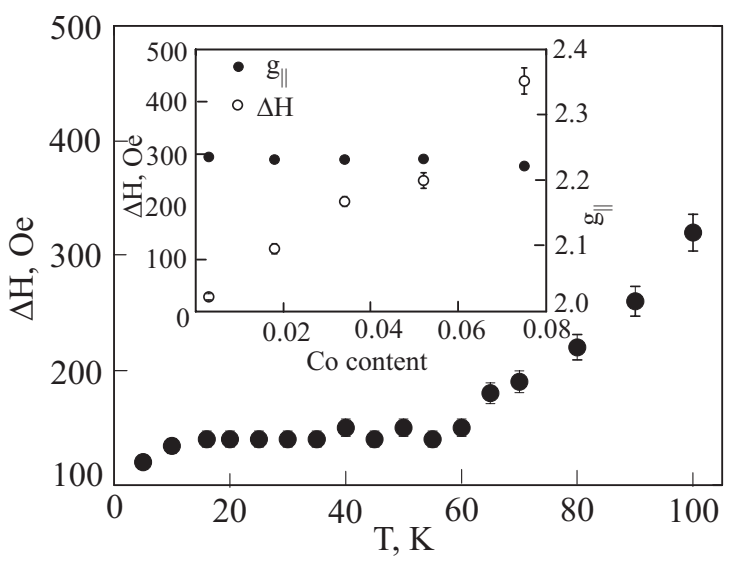

Fig. 3. Temperature dependence of the EPR linewidth for $x=0.018$. The inset shows $\Delta H$ (the left scale) and $g_{\|}$(the right scale) in a function of the Co content.
$\Delta H$ decreases as the temperature is decreased from $100 \mathrm{~K}$ to $60 \mathrm{~K}$ and remains almost constant at temperatures below $\sim 60 \mathrm{~K}$ down to $4 \mathrm{~K}$.

The fast decrease of $\Delta H(T)$ can be naturally explained by the temperature dependence of the spin lattice relaxation time of $\mathrm{Co}^{2+}$ ions, since it is observed even at lowest $x$. However, the "plateau» below $60 \mathrm{~K}$ has clearly a magnetic origin. As we mentioned above, according to previously reported EPR data on Mn-doped DMS, one would expect that $\Delta H$ increases as the temperature is decreased due to the fluctuations of antiferromagnetically coupled TM ions. Therefore the independence of $\Delta H(T)$ on temperature can be interpreted as the absence of any such coupling between $\mathrm{Co}^{2+}$ contributing to the observed line.

It is clear that the exchange narrowing scenario is rather unlikely in $\mathrm{ZnO}$ :Co, since simple estimates of $\Delta H \sim x M_{2} / J$, in the case where the second moment $M_{2}$, stems from the magnetic dipole or Dzyaloshinskii-Moriya interaction, lead to a negligible linewidth, which is about two orders of magnitude smaller than the observed one.

The situation changes, however, if one considers $M_{2}$ arising from the single ion anisotropy $\mathcal{H}_{A}$. In this case $\Delta H \sim x(2 D)^{2} / J$ gives a linewidth of several kOe i.e. much larger then the observed one. This is an indication that $\mathcal{H}_{A}$ of $\mathrm{Co}^{2+}$ should be included in the unperturbed Hamiltonian $\mathcal{H}_{0}$ which determines the structure of the energy levels between which the EPR absorption takes place. Here the exchange modulation of a broadened line cannot be effective, because $\mathcal{H}_{A}$ does not commute with $\mathcal{H}_{\text {ex }}$.

Kopvillem has developed a theory of the second moment in the case where $\mathcal{H}_{0}$ includes the single ion anisotropy and Zeeman terms $\mathcal{H}_{Z}$ while the exchange and the dipole interactions were considered as perturbations [31]. The underlying physical picture is the following. The $\mathcal{H}_{A}$ and $\mathcal{H}_{Z}$ split the energy spectrum of the spin system into a series of quasi-discrete bands whose widths depend on the magnetic dipole and exchange interactions among the paramagnetic centers. In its turn the EPR spectrum splits up into a series of fine-structure components corresponding to the magnetic dipole transitions $\left|S, m_{S}\right\rangle \leftrightarrow\left|S, m_{S} \pm 1\right\rangle$. Under these conditions the second moment is given by

$$
M_{2}=\sum_{k}\left[b_{j k}^{2} P_{1}+b_{j k} J_{j k} P_{2}+J_{j k}^{2} P_{3}\right],
$$

where the summation is over all lattice sites, $b_{j k}=$ $=(3 / 2) \mu_{B}^{2} g_{\|}^{2}\left(1-3 \cos ^{2}\left(\theta_{j k}\right)\right) / r_{j k}^{3}, J_{j k}$ are the exchange constants and $P_{i}$ are numerical constants having the following values in the case of $S=3 / 2$ and the transition between $m_{S}= \pm 1 / 2: P_{1}=1.125, P_{2}=-3 / 4, P_{3}=3 / 8$.

The above formula clearly indicates that the exchange and magnetic dipole interactions are responsible for a linewidth broadening but it cannot be employed directly to calculate the linewidth in $\mathrm{ZnO}$ : $\mathrm{Co}$ for the following 
reasons. As was pointed out by Kittel and Abrahams, in the case of very diluted paramagnets $(x<<0.1)$, when only the magnetic dipole interaction is taken into account, the ratio $M_{4} / M_{2}^{2} \gg 1$ (where $M_{4}$ is the fourth moment) and a resonance line is better described by a cut-off Lorentzian line with $\Delta H \ll M_{2}^{1 / 2}$ [32]. The summation in the $M_{2}$ and $M_{4}$ formulae, which have to be carried out over the occupied sites only, is replaced by the sum over all crystal sites so that both moments are proportional to the concentration of magnetic ions, as well as the linewidth $\Delta H \simeq x M_{2}^{3 / 2} / M_{4}^{1 / 2}$.

To adapt this approach to the case treated in [31] one needs an estimate of $M_{4}$. Let us first note that the second moment (2) is a sum of pair contributions coming from the dipole and exchange interactions between different $\mathrm{Co}^{2+}$ sites. It is easy to show that with the choice of $\mathcal{H}_{0}$ made above the perturbation Hamiltonians, $\mathcal{H}_{\mathrm{ex}}$ and $\mathcal{H}_{d}$, will cause additional transitions at frequencies $v_{0} \pm\left(p J_{j k}+q b_{j k}\right)$, where $v_{0}$ is the unperturbed frequency and $p$ and $q$ are constants of the order of unity. Thus $M_{2}$ is simply proportional to $\sum_{k}\left(p J_{j k}+q b_{j k}\right)^{2}$ in agreement with Kopvillem's formula. Quite similarly the leading term in $M_{4}$, which is proportional to $x$, can be written as $\sum_{k}\left(p J_{j k}+q b_{j k}\right)^{4}$. Using these estimates one can recover a linear dependence of the linewidth on the magnetic ion concentration, $\Delta H \sim x M_{2}^{1 / 2}$, which is in qualitative agreement with our experimental data.

Another interesting point is the role played by the exchange interaction in the mechanism of linewidth formation. As we explained above, a pair of $\mathrm{Co}^{2+}$ spins coupled by the exchange constant $J$ contributes to EPR spectrum at frequencies (or magnetic fields) which are proportional to this particular value of $J$. Hence if one puts in (2), for example, $J \simeq 10 \mathrm{~K}$ (an estimate for the nearest neighbor exchange integral in $\mathrm{ZnO}: \mathrm{Co}) \Delta H$ of the order of several teslas will be obtained, which is certainly meaningless. Therefore we should restrict the sum to those pair contributions which do not exceed the experimentally observed $\Delta H$.

Some guidelines on how to do this provide our pair spectrum presented in Fig. 2,b. In fact the exchange-pair transitions shown in this figure are both involved in the main resonance line broadening, but the ones with $J>0.0295 \mathrm{~cm}^{-1}$, which are too far in the wings, do not contribute to the observed linewidth. One can argue, by comparing the exchange constants obtained in this work with those found for $\mathrm{ZnO}: \mathrm{Mn}$ [33], that in the case of $J=0.0295 \mathrm{~cm}^{-1}$ we are dealing with fifth or sixth neighbor in the $\mathrm{ZnO}$ lattice. Thus, we have arrived at an important conclusion that a large number of $\mathrm{Co}^{2+}$ neighbors in $\mathrm{ZnO}$ lattice $(n \leq 5)$ have no effect on the observed linewidth. This could bring an explanation for the experimentally established absence of strong antiferromagnetic fluctuations at low temperatures in $\mathrm{ZnO}: \mathrm{Co}$.
In summary, we have reported the $X$-band EPR studies of single crystalline $\mathrm{Zn}_{1-x} \mathrm{Co}_{x} \mathrm{O}(x=0.001-0.075)$ thin films grown by plasma-assisted molecular beam epitaxy. By analyzing the EPR linewidth behavior, we show that a combined effect of the exchange and dipolar broadening plays an important role in the mechanism of linewidth formation in this material.

The authors thank C. Deparis and C. Morhain for the sample preparation. Two of us (V.P. and A.S.) dedicate this article to the memory of their dear mentor, Anatolii Illarionovich Zvyagin, an eminent scientist and wonderful man.

1. H. Ohno, Science 281, 951 (1998).

2. Y. Ohno, D.K. Young, B. Beschoten, F. Matsukura, H. Ohno, and D.D. Awschalom, Nature 402, 790 (1999); T. Dietl, H. Ohno, F. Matsukura, J. Cibert, and D. Ferrand, Science 287, 1019 (2000).

3. K. Sato and H. Katayama-Yoshida, Physica E10, 251 (2001).

4. C. Song, K.W. Geng, F. Zeng, X.B. Wang, Y.X. Shen, F. Pan, Y.N. Xie, T. Liu, H.T. Zhou, and Z. Fan, Phys. Rev. B73, 024405 (2006).

5. M. Venkatesan, C.B. Fitzgerald, J.G. Lunney, and J.M.D. Coey, Phys. Rev. Lett. 93, 177206 (2004).

6. J.R. Neal, A.J. Behan, R.M. Ibrahim, H.J. Blythe, M. Ziese, A.M. Fox, and G.A. Gehring, Phys. Rev. Lett. 96, 197208 (2006).

7. A.C. Tuan, J.D. Bryan, A.B. Pakhomov, V. Shutthanandan, S. Thevuthasan, D.E. McCready, D. Gaspar, M.H. Engelhard, J.W. Rogers, Jr., K. Krishnan, D.R. Gamelin and S.A. Chambers, Phys. Rev. B70, 054424 (2004).

8. K.R. Kittistved, N.S. Norberg, and D.R. Gamelin, Phys. Rev. Lett. 94, 147209 (2005).

9. M.H. Kane, K. Shalini, C.J. Summers, R. Varatharajan, J. Nause, C.R. Vestal, Z.J. Zhang, and I.T. Ferguson, J. Appl. Phys. 97, 023906 (2005).

10. S. Yin, M.X. Xu, L. Yang, J.F. Liu, H. Rosner, H. Hahn, H. Gleiter, D. Schild, S. Doyle, T. Liu, T.D. Hu, E. Takayama-Muromachi, and J.Z. Jiang, Phys. Rev. B73, 224408 (2006).

11. J. Alaria, H. Bieber, S. Colis, G. Schmerber, and A. Dinia, Appl. Phys. Lett. 88, 112503 (2006).

12. M. Bouloudenine, N. Viart, S. Colis, J. Kortus and A. Dinia, Appl. Phys. Lett. 87, 052501 (2005).

13. S. Kolesnik, B. Dabrowski, and J. Mais, J. Appl. Phys. 95, 2582 (2004).

14. Jae Hyun Kim, Hyojin Kim, Dojin Kim, Soon Gil Yoon, and Woong Kil Choo, Solid State Commun. 131, 677 (2004).

15. S-J. Han, B.Y. Lee, J.-S. Ku, Y.B. Kim, and Y.H. Jeong, J. Magn. Magn. Mater. 272, 2008 (2004).

16. A.S. Risbud, N.A. Spaldin, Z.Q. Chen, S. Stemmer, and R. Seshadri, Phys. Rev. B68, 205202 (2003).

17. T. Chanier, M. Sargolzaei, I. Opahle, R. Hayn, and K. Koepernik, Phys. Rev. B73, 134418 (2006).

18. E.C. Lee and K.J. Chang, Phys. Rev. B69, 085205 (2004).

19. M.H.F. Sluiter, Y. Kawazoe, P. Sharma, A. Inoue, A.R. Raju, C. Rout, and U.V. Waghmare, Phys. Rev. Lett 94, 187204 (2005). 
20. A. Abragam and B. Bleaney, Electron Paramagnetic Resonance of Transition Ions, Clarendon, Oxford, (1970).

21. N. Samarth and J.K. Furdyna, Phys. Rev. B37, 9227 (1988).

22. S.B. Oseroff, Phys. Rev. B25, 6584 (1982).

23. H.A. Sayad and S.M. Bhagat, Phys. Rev. B31, 591 (1985).

24. R.E. Kremer and J.K. Furdyna, Phys. Rev. B31, 1 (1985).

25. E. Chikoidze, H.J. von Bardeleben, Y. Dumont, P. Galtier, and J.L. Cantin, J. Appl. Phys. 97 10D327 (2005).

26. A. Ben Mahmoud, H.J von Bardeleben, J.L. Cantin, A. Mauger, E. Chikoidze, and Y. Dumont, Phys. Rev. B74, 115203 (2006)
27. P. Sati, R. Hayn, R. Kuzian, S. Regnier, S. Schafer, A Stepanov, C. Morhain, C. Deparis, M. Laugt, M. Goiran, and Z. Golacki, Phys. Rev. Lett. 96, 017203 (2006).

28. P. Sati, C. Deparis, C. Morhain, S. Schafer, and A. Stepanov, Phys. Rev. Lett. 98, 137204 (2007).

29. T. Estle and M. De Wit, Bull. Am. Phys. Soc. 6, 445 (1961).

30. N. Jedrecy, H.J. von Bardeleben, Y. Zheng, and J-L. Cantin, Phys. Rev. B69, 041308(R) (2004).

31. U.Kh. Kopvillem, Soviet Phys. JETP 11, 109 (1960).

32. C. Kittel and E. Abrahams, Phys. Rev. 90, 238 (1953).

33. X. Gratens, V. Bindilatti, N.F. Oliveira, Jr., Y. Shapira, S. Foner, Z. Golacki, and T.E. Haas, Phys. Rev. B69, 125209 (2004). 